\title{
WIND-INDUCED RESPONSE ANALYSIS OF THE CYLINDRICAL RETICULATED MEGA-STRUCTURES
}

\author{
Yongjun $\mathrm{He}^{1, *}$, Xuhong Zhou ${ }^{1,2}$ and Haishun Wang ${ }^{3}$ \\ ${ }^{1}$ Professor, College of Civil Engineering of Hunan University, Changsha 410082, P.R. China \\ ${ }^{2}$ Professor, College of Civil Engineering of Chongqing University, Chongqing 400044, P.R. China \\ ${ }^{3}$ graduate student, College of Civil Engineering of Hunan University, Changsha 410082, P.R. China \\ *(Corresponding author: E-mail: hyj0087@163.com)
}

Received: 15 April 2015; Revised: 1 June 2015; Accepted: 18 June 2015

\begin{abstract}
With the further increase of span of spatial structures, wind load becomes one of the governing factors in structural design. Attention is paid to wind-induced response analysis of spatial grid structures in the recent years. Research on the wind-induced responses of the cylindrical reticulated mega-structure with double-layer plane-plate grid substructures is conducted by time-history method in this paper. The basic distribution laws of the wind-induced member internal forces and nodal displacements of both the main structure and substructures are first analyzed. By detailed parametric analyses, the effect of the geometric parameters, damping ratio, load, boundary condition, and wind velocity on structural wind-induced response is then studied. Finally the structural wind-induced vibration coefficients are analyzed, and their rational values are recommended. All this work will provide theoretical guidance to the wind-resistance design of this kind of structure.
\end{abstract}

Keywords: Cylindrical reticulated mega-structure, Time-history method, Wind-induced response, Parametric analyses, Wind-induced vibration coefficient

DOI: $10.18057 /$ IJASC.2016.12.1.6

\section{INTRODUCTION}

With the development of human society, people have put forward higher requirement for the span of spatial structures (Makowski [1]). Double-layer cylindrical reticulated structure is one kind of long span structures, which is used extensively. However, with further increase of the span, the double-layer cylindrical reticulated structure will meet some inevitable problems, such as the instability of the whole structure since the thickness-to-span ratio is too small, the buckling of struts for the members are too long and the internal forces are too large, as well as the increasing steel consumption and the corresponding economic problems. To break the restriction of large internal forces of members and structural overall instability to construct super-long-span structure, the concept of reticulated mega-structure has been proposed (He and Zhou [2]). The whole structure is made up of two levels. The first level is the large grid structure called the main structure consisting of the transverse and longitudinal latticed 3D beams, which bears all the loads and transfers them to the supporting structure. The second level is composed of substructures embedded in the large grids of the main structure, which only bears loads within the corresponding large grids and transfer them to the main structure. The structural configuration, support styles, mechanical model, static properties, and stabilities of this kind of structure have been comprehensively investigated (He and Zhou [2-3]).

Generally, the spatial structures are comparatively sensitive to wind for their long span and light weight (Lu et al. [4]). Thus, wind load is one of the governing factors in structural design. However, the research findings on wind-resistance of spatial structures are still inadequate, and the design parameters for wind-resistance of spatial structures are not perfect in the current design code. They are commonly determined by referring to those of the high-rise buildings (Mehta [5]), and this is 
obviously unreasonable. Therefore, attention should be paid to the reasonable analysis and calculation of wind-induced response in design of long-span spatial structures (Chen et al. [6]). For the reticulated mega-structure, as an innovative long-span spatial structure, its wind-resistant performance is still obscure. Therefore, this paper is focused on research of the wind-induced response of the cylindrical intersecting-latticed-three-dimensional-beams-system (ILTDBS) reticulated mega-structure with double-layer plane-plate grid substructures as shown in Figure 1.

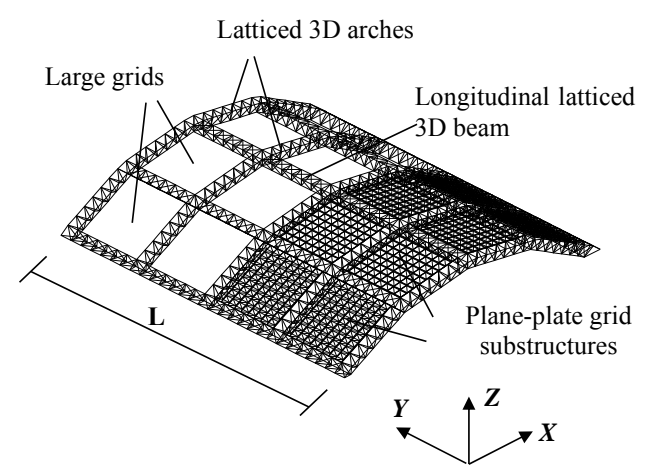

(a) Three-dimensional configuration

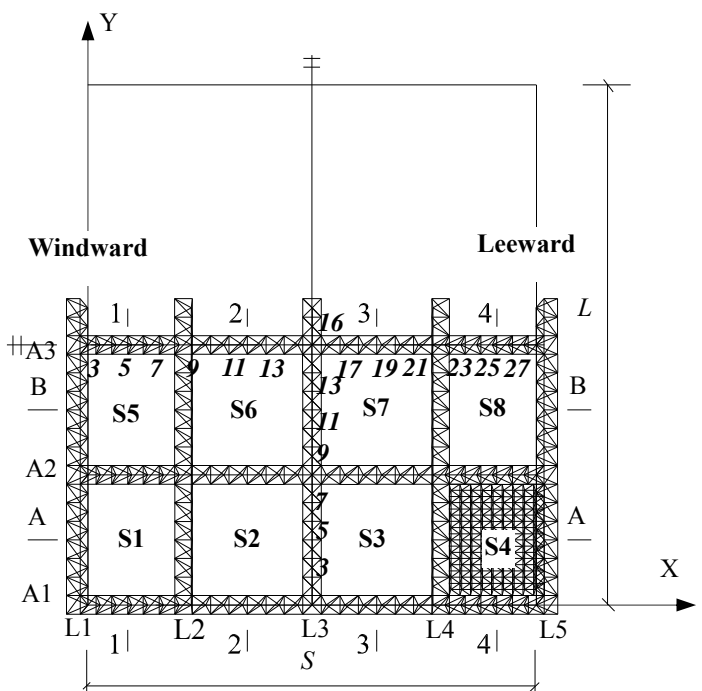

(c) The plan of the structure with numbering of the substructures, latticed 3D arches, longitudinal beams, members, and the nodes

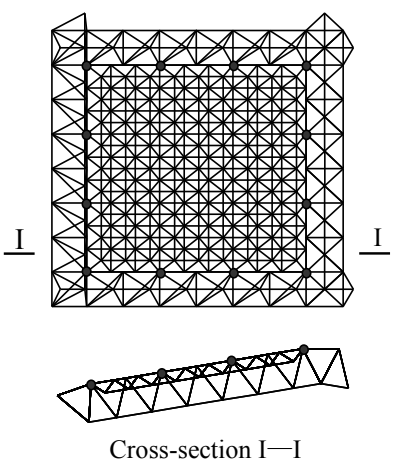

(b) Substructure embedded in large grid

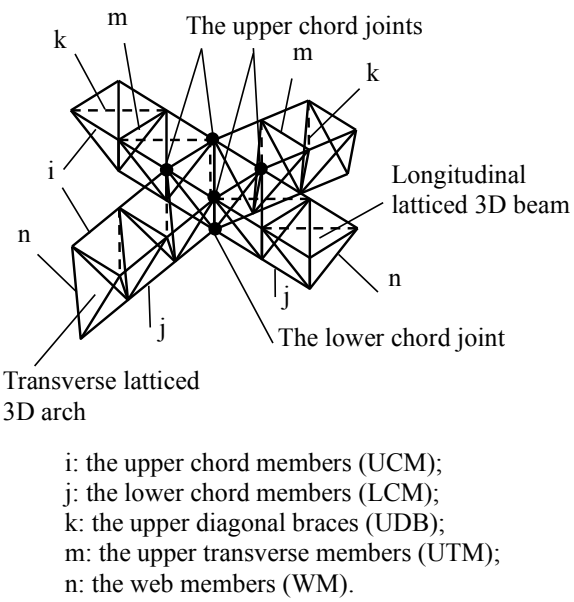

(d) Main node of the main structure, i.e. the intersection area of the latticed 3D beams

Figure 1. Cylindrical ILTDBS Reticulated Mega-structure with Double-layer Plane-plate Grid Substructures

\section{ANALYTICAL METHOD OF WIND-INDUCED RESPONSE}

The calculation methods of structural wind-induced response can be divided into two categories, i.e., the frequency domain method and time history method (Oskoei and McClure [7]). The former is to solve the structural wind-induced response from power spectrum, and it can objectively reflect the regularity of strong wind (Soares and Mansur [8]). The calculation complexity of the frequency domain method is much less than that of the time history method, because it is to directly calculate the statistical result of random response in frequency domain. Nevertheless, it is difficult to determine the number of vibration modes accurately for the long span space grid structures 
(Uematsua et al. [9]). For the time history method, the dynamic equilibrium equation is directly integrated according to the adopted wind velocity spectrum, and the nodal displacement, velocity as well as the acceleration at various moments under wind load can be calculated by step-by-step integration (Thai and Kim [10]). The stochastic simulation time history method (Zhang [12] and Masters et al. [13]), an effective analysis method for response of nonlinear structures under stochastic dynamic load, is adopted in this paper to study the structural wind-induced response characteristics. The analysis process can be described as follows.

(1) According to the statistical characteristics of wind load, the artificial wind velocity time history series (excitation samples) with specified spectral density and spatial correlation are generated and converted into wind pressure time history series applied on structure (Karmakar et al. [14]). And the improved AR (Auto-Regressive) method is adopted to realize the numerical simulation of random wind load with consideration of temporal and spatial correlation (Owen et al. [15]).

(2) The structural dynamic equilibrium equation under the stochastic wind load $P(t)$ is established by finite element method, which can be expressed as

$$
[M]\{\ddot{U}\}+[C]\{\dot{U}\}+[K]\{U\}=\{P(t)\}
$$

in which, $[M],[C]$, and $[K]$ are the mass, Rayleigh damping [11], and stiffness matrices,

respectively; $\{U\},\{\dot{U}\},\{\ddot{U}\}$, and $\{P(t)\}$ are the nodal displacement, velocity, acceleration, and the wind load vectors, respectively. The Newmark- $\beta$ step-by-step integration method (Soares and Mansur [16]) is then adopted to solve the dynamic equation in time domain, and the structural geometrical nonlinearity is considered by Newton-Raphson iterative technique (Kim et al. [17]) to obtain the structural wind-induced response.

(3) Statistical analysis on the response samples is carried out to obtain the required statistical information, such as the means and standard deviations of the nodal displacements, velocities, accelerations and those of the member internal forces, as well as the corresponding spectrum characters. And the structural wind-induced response characteristics can thus be determined.

The Davenport's power spectrum (Davenport [18] and Iwatani [19]) is adopted to simulate the wind velocity time history series of various nodes of the structure with consideration of temporal and spatial correlation by AR model (Isyumov [20]). The corresponding computational program for simulation of stochastic wind field and analysis of structural wind-induced response is developed. Numerical examples indicate that this method is feasible and highly efficient for analysis of wind-induced response of long span spatial structures. Theoretically, this method can be adopted for any system and excitation. Also, the whole process information of structural dynamic response can be obtained.

\section{BASIC WIND-INDUCED RESPONSE CHARACTERS OF THE RETICULATED MEGA-STRUCTURE}

\subsection{Analytical Model}

Take the cylindrical reticulated mega-structure with span of $80 \mathrm{~m}$ to do analysis. The basic model and concerned parameters can be described as follows. The ratio of rise to span of the main structure $F=0.2$, height of the latticed 3D beams is $3.6 \mathrm{~m}$, numbers of large grids of the main structure is $4 \times 4$, and the number of upper chord small grids within a latticed 3D beam segment 
$n=6$, The double-layer plane-plate grid substructures are imbedded in the main structure by perimeter-points support style as shown in Figure 1(b), the number of the upper chord small grids of each substructure is $9 \times 9$. The whole structure consists of steel round tube members, and the members are connected by welded spherical joints. The cross section of members in the main structure $\left(S_{\mathrm{m}}\right)$ and that in the substructures $\left(S_{\mathrm{s}}\right)$ are $S_{\mathrm{m}}=\varphi 140 \times 4.5$ and $S_{\mathrm{s}}=\varphi 76 \times 4.0$, respectively. The yield stress of the steel is $235 \mathrm{MPa}$ and the damping ratio is taken as 0.02 . The fixed-hinged supports are set on the upper and lower chord joints of the main nodes along the two longitudinal edges of the main structure as described in Reference (He and Zhou [2]), and the vertical uniform loads of $1 \mathrm{kN} / \mathrm{m}^{2}$ are applied on the upper surface of the structure. The plan of the structure with numbering of some members is shown in Figure 1(c). In the figure, A1-A3, L1-L5, and S1-S8 denote the numberings of the latticed 3D arches, longitudinal latticed 3D beams, and the substructures, respectively. Only half of the structure is displayed for its symmetry. Also, not all the substructures braced in the large grids are shown in the figure for clarity.

The basic wind velocity under the standard condition, i.e. the 10 -minute mean wind velocity at a height of $10 \mathrm{~m}$ above the ground, is taken as $25 \mathrm{~m} / \mathrm{s}$, and the direction of the wind is along the $\mathrm{x}$-axis as shown in Figure 1. The air density is $1.225 \mathrm{~kg} / \mathrm{m}^{3}$. And the terrain roughness of C-class is considered. Obviously, the number of samples increases with increase of the wind duration in wind-induced response analysis, and thus the precision of the statistical result of the response increases. However, the increase of wind duration will inevitably result in decline of the calculation efficiency. Therefore, giving consideration to both the precision and calculation efficiency as discussed in detail in our previous work (He et al. [21]), the wind duration and time step in analysis are taken as $120 \mathrm{~s}$ and $0.1 \mathrm{~s}$, respectively.

As we know, the wind load is composed of two parts, i.e., the mean and fluctuating ones. The average value of the structural response reflects the action effect of mean wind (the equivalent static wind) to a certain degree, whereas the standard deviation indicates the fluctuating effect of wind. The standard deviation of the structural response under the fluctuating wind velocity with a certain assurance rate is usually taken as the design value of fluctuating response in practical engineering (Jakobsen [22]). Thus the distribution and variation laws of means and standard deviations of the structural responses are taken as the main indices in analysis. Also, the calculated result shows that, the longitudinal (y-directional) displacement component of any node of the structure is extremely small in comparison with the transverse (x-directional) and vertical (z-directional) ones. Therefore, this paper is focused on the transverse and vertical nodal displacements in research of the structural displacement response characteristics.

\subsection{Wind-induced Response of the Main Structure}

\subsubsection{Internal force response characteristics}

From the calculated results (the concrete data are not listed), it is found that the wind-induced internal forces of various members of the latticed 3D arches at both ends of the structure are relatively smaller than those of the corresponding members of various middle latticed 3D arches. The internal force distribution laws of various latticed 3D arches are approximately identical. Therefore, the middle latticed 3D arch A3 of the structure is typically taken to study in this paper. The internal force means (IFM) and standard deviations (IFSD) of various types of members in the latticed 3D arch are displayed in Figures 2 and 3, respectively. In the figures, UCM, LCM, UDB, UTM, and WM denote the upper and lower chord members, upper diagonal braces, upper transverse members, and the web members, respectively, as shown in Figure 1(d), and the same as the following figures. 


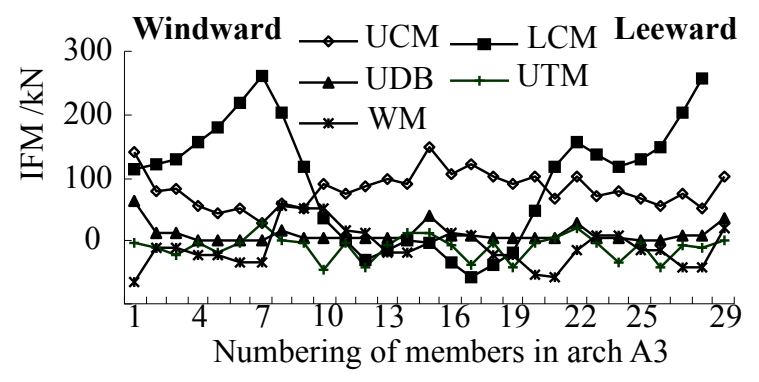

Figure 2. Distribution of the Internal Force Means (IFM) of Various Members in the Latticed 3D Arch A3

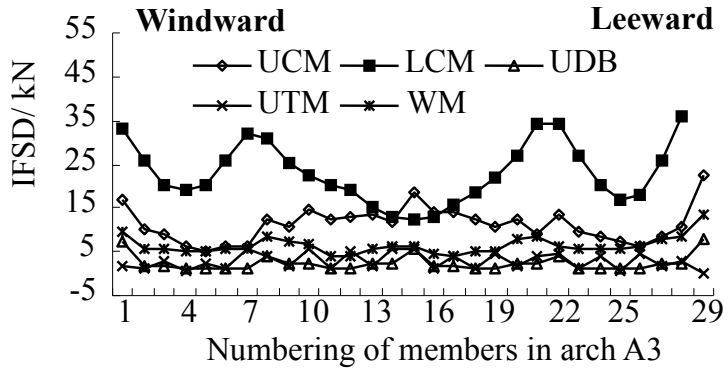

Figure 3. Distribution of the Internal Force Standard Deviations (IFSD) of Various Members in the Latticed 3D Arch A3

It can be seen that, the internal forces of the upper and lower chord members are comparatively large and thus they are the main load-bearing components. The maximum wind-induced internal forces exist in the lower chord members at the bottom of the leeward side of the latticed 3D arch. The wind-induced internal forces of various types of members basically show symmetrical distribution with respect to the top of the arch. The internal forces of various types of members all fluctuate along the arch and the local extrema emerge in the intersection areas of the transverse latticed 3D arch with various longitudinal latticed 3D beams. The internal force fluctuation range of the lower chord members is very large. The internal forces of the lower chord members at the middle of the windward side and the bottom of the leeward side of the latticed 3D arch are comparatively large, while those on the top of the arch are relatively small. The internal force fluctuation range of the upper chord members is slightly smaller than that of the lower chord members, and the internal forces nearly present $\mathrm{W}$-shaped distribution along the latticed 3D arch with comparatively large values at the top and bottoms of both sides of the arch. The upward suction force of wind on the leeward side leads to increase of the tensile forces of the upper chord members and decrease of those of the lower chord members. This is the main reason for difference of the internal force distribution law between the upper and lower chord members. The internal forces of the web members, upper diagonal braces and the transverse members are relatively small. The fluctuation laws of them are not remarkable and the fluctuation ranges are very small.

Calculated results show that, the wind-induced internal forces of the upper and lower chord members of various longitudinal latticed 3D beams are greatly smaller than those of the latticed 3D arches. The internal forces of members of the top latticed 3D beam L3 are obviously larger than those of the corresponding members of the middle and lower latticed 3D beams. However, the internal force distribution laws of various parallel longitudinal latticed 3D beams are approximately identical and show symmetrical distribution with respect to the midpoints of the latticed 3D beams. Therefore, one half of the top latticed 3D beam L3 is taken to study, and the internal force means and standard deviations of various types of members in the latticed 3D beam are displayed in Figures 4 and 5, respectively.

Obviously, the largest internal force of the longitudinal latticed 3D beam exists in the upper transverse members. The internal forces of the upper transverse members fluctuate along the axial direction of the longitudinal latticed 3D beam with local extrema in the intersection areas with various transverse arches. Also, the internal forces of the lower chord members in the middle of the end segment (i.e., near by the Section A-A as shown in Figure 4(c)) are comparatively large, and there is not notable fluctuation in other place. The reason is that the constraint of the end latticed 3D arch on the longitudinal latticed 3D beams is comparatively weak, which increases the local 
wind-induced vibration effect of the longitudinal latticed 3D beams in these places. The internal forces and their fluctuation ranges of other types of members are comparatively small.

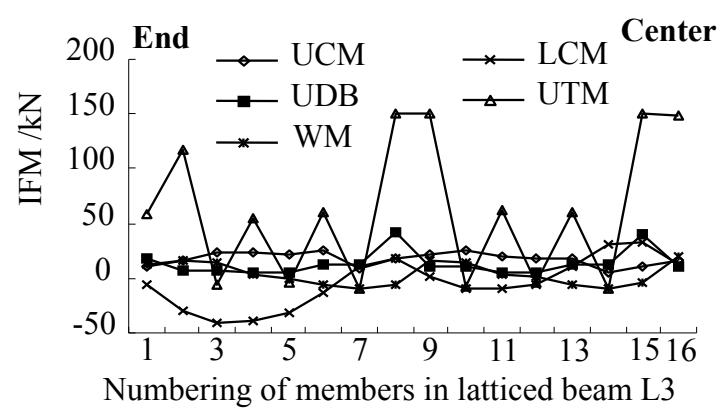

Figure 4. Distribution of IFM of Various

Members in the Longitudinal Latticed 3D Beam L3

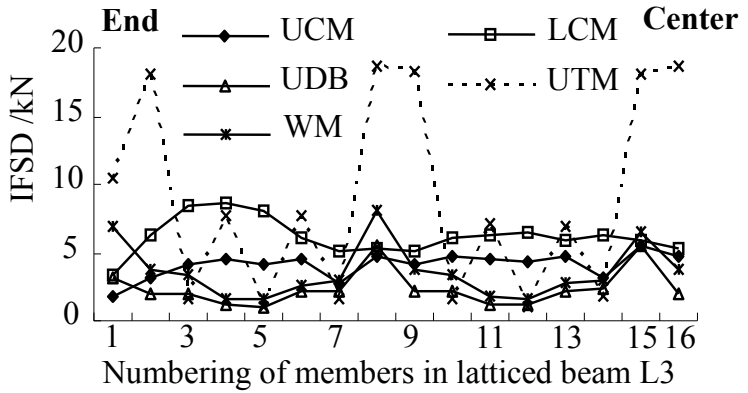

Figure 5. Distribution of IFSD of Various Members in the Longitudinal Latticed 3D Beam

\subsubsection{Displacement response characteristics}

From the calculated results (the concrete data are not listed) it can be seen that, the nodal displacement distribution laws of various latticed 3D arches are basically identical expect that the nodal displacements of various middle arches are slightly larger than those of the both end arches. Moreover, the nodal displacement distribution laws of various parallel longitudinal latticed 3D beams are also approximately identical. However, for various parallel longitudinal latticed 3D beams, the largest upper chord nodal displacement exists in the latticed 3D beam L4 located in the middle of the leeward side of the structure; while the largest lower chord nodal displacement exists in the latticed 3D beam L2 in the middle of the windward side. Here, the middle latticed 3D arch A3 and top longitudinal latticed 3D beam L3 are typically taken to study the nodal displacement distribution law of the structure in detail. The means and standard deviations of the horizontal and vertical displacements of various nodes of the latticed 3D arch A3 and beam L3 are shown in Figs. $6,7,8$, and 9, respectively. In the figures, $\mathrm{UH}, \mathrm{UV}, \mathrm{LH}$, and $\mathrm{LV}$ present the horizontal and vertical displacements of the upper chord nodes and those of the lower chord nodes, respectively; the numbering of nodes is identical with that of the corresponding members as shown in Figure 1(c); and the same as the following figures.

As shown in Figures 6 and 7, the vertical displacements of both the upper and lower chord nodes are larger than the horizontal ones, and the nodal displacement increases gradually from the bottom to top of the arch. Therefore, the vertical displacement of the node on the top of the latticed 3D arch is the major governing factor in wind-resistance design. It can be seen from Figures 8 and 9 that, the nodal displacements of the longitudinal latticed 3D beam show symmetrical distribution with respect to the midpoint of the latticed 3D beam, and the vertical component of each is greatly larger than the corresponding horizontal one. The vertical displacements of the upper chord nodes are basically consistent with those of the corresponding lower chord nodes, while the horizontal displacements of the lower chord nodes are slightly larger than those of the corresponding upper chord nodes. The vertical displacements of the nodes at the end of the longitudinal latticed 3D beam are slightly smaller than those of the others in it, but the different in horizontal displacements of various nodes is very small. In a word, for the longitudinal latticed 3D beam, the nodal displacement fluctuation along its longitudinal axis is not remarkable, and the vertical displacement is the major governing factor in design. 


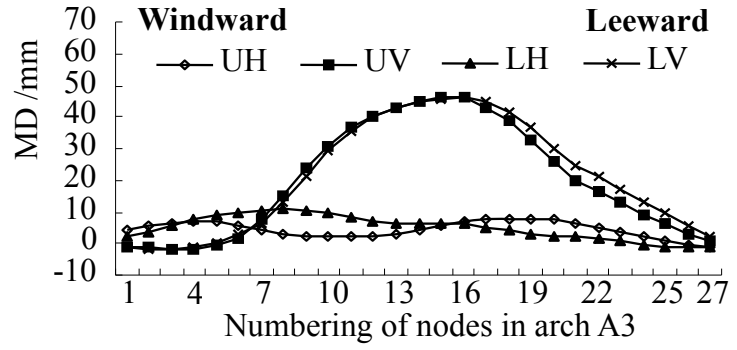

Figure 6. The Displacement Means (DM) of Various Nodes in the Latticed 3D Arch

A3

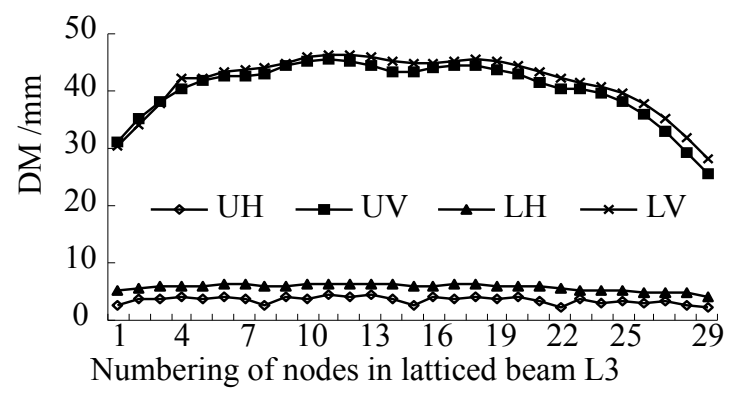

Figure 8. The DM of Various Nodes in the Longitudinal Latticed 3D Beam L3

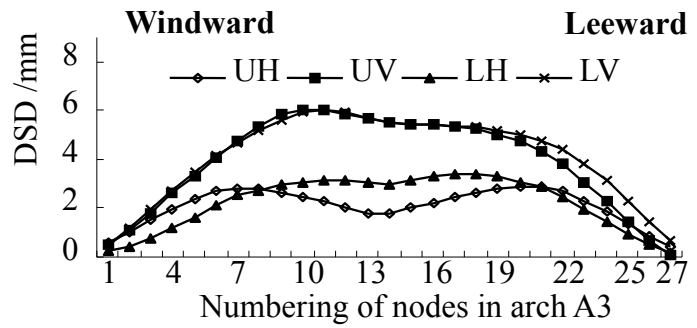

Figure 7. The Displacement Standard Deviations (DSD) of Various Nodes in the Latticed 3D Arch A3

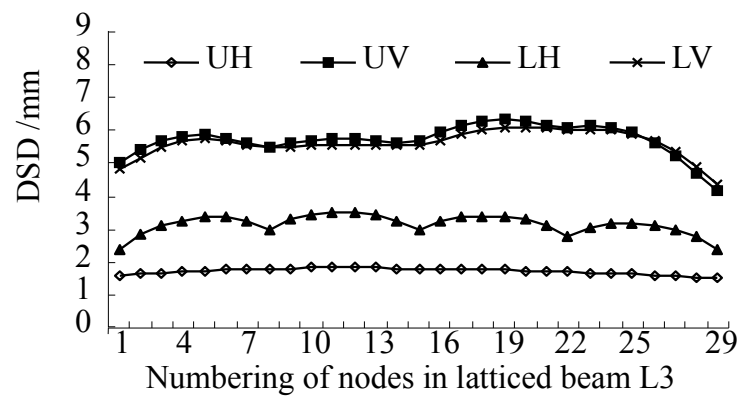

Figure 9. The DSD of Various Nodes in the Longitudinal Latticed 3D Beam L3

\subsection{Wind-induced Response of the Substructures}

\subsubsection{Internal force response characteristics}

Figures 10 and 11 depict the distributions of the wind-induced internal force means and standard deviations of various members in the substructures along the transverse section B-B. It can be seen that the internal forces of the transverse (x-directional) upper and lower chord members (TUCM and TLCM) in the upper substructure are obviously larger than those in the lower substructure. And the member internal forces of the lower substructure on the leeward side are larger than those on the windward side. The internal forces of the upper chord members are larger than those of the lower chord and other types of members, thus the upper chord members are the main load-bearing components. Also, the internal forces of the transverse upper chord members are obviously larger than those of the longitudinal (y-directional) members (LUCM). The internal force variation range of the web members is small, so these members can be designed according to the constructional requirement. The member internal force distribution laws of various substructures along the transverse direction are basically identical. That is, the closer to the center of the substructures, the larger the internal forces of the members are, except the internal forces of the web members, which scarcely fluctuate.

From Figures 12 and 13 it can be seen that, the member internal force distribution laws of various substructures with same height along the longitudinal direction are nearly identical. Moreover, the distribution laws of the fluctuating wind-induced internal forces are close to those of the mean internal forces. The internal force distribution laws of the longitudinal upper and lower chord members along the longitudinal direction are similar to those of transverse chord members along the transverse direction as shown in Figures 10 and 11, so they are not depicted again in here. Additionally, the internal forces of the transverse upper chord members basically do not fluctuate along the longitudinal direction. 


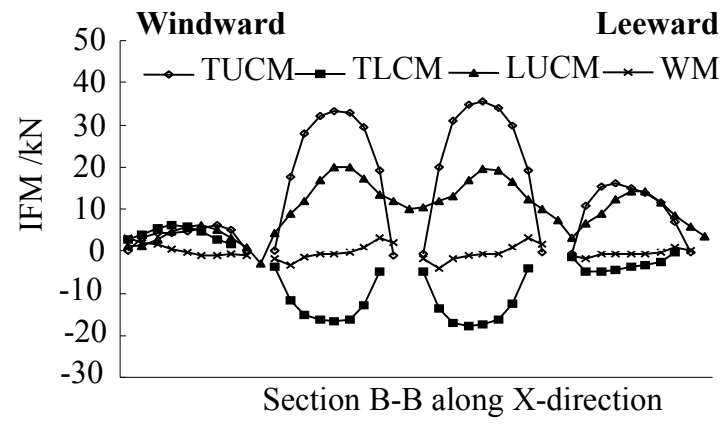

Figure 10. The IFM of Various Members in the Substructures along Section B-B

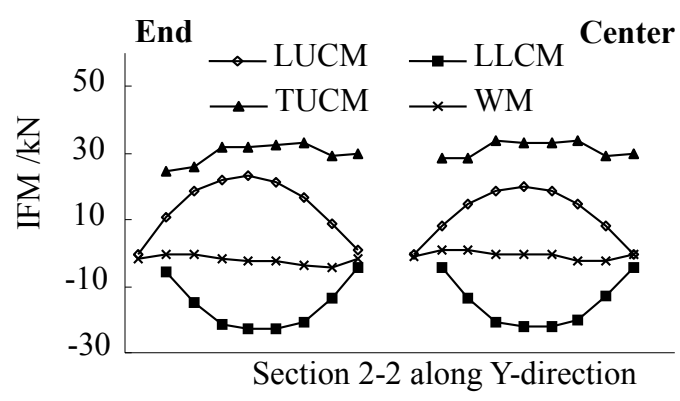

Figure 12. The IFM of Various Members in the Substructures along Section 2-2

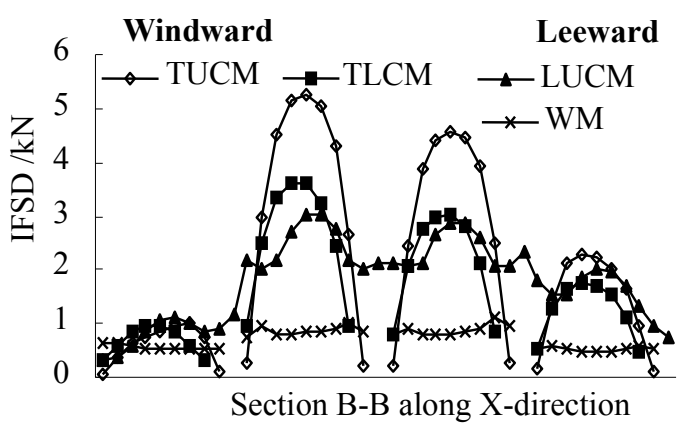

Figure 11. The IFSD of Various Members in the Substructures along Section B-B

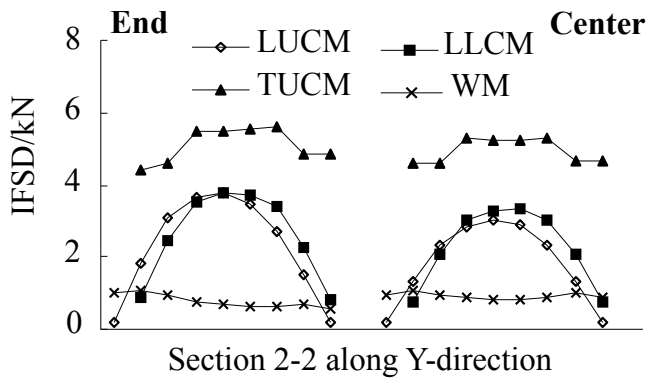

Figure 13. The IFSD of Various Members in the Substructures along Section 2-2

\subsubsection{Displacement response characteristics}

Figures 14 and 15 depict the variation of the means and standard deviations of wind-induced nodal displacements of the substructures along the section B-B, respectively. It can be seen that, under the action of mean wind, there is not remarkable difference in the nodal transverse horizontal displacements between various substructures, while the difference in the nodal vertical displacements is notable. The nodal displacements of the upper substructures are larger than those of the lower substructures, and the displacements of the nodes on the leeward side are slightly larger than those on the windward side. The nodal vertical displacements of upper substructures are greatly larger than the horizontal ones. Under fluctuating wind load, the nodal displacement response of various substructures basically shows symmetrical distribution with respect to the top of the structure. The nodal displacements of the upper substructures are larger than those of the lower substructures. Additionally, the nodal vertical displacements are obviously larger than the horizontal ones, this indicates that the wind-induced vibration of the substructures is mainly along the vertical direction. Also, the nodal displacements of the lower substructures increase gradually from the bottom up, while those of each upper substructure decrease gradually from the center to both sides. 


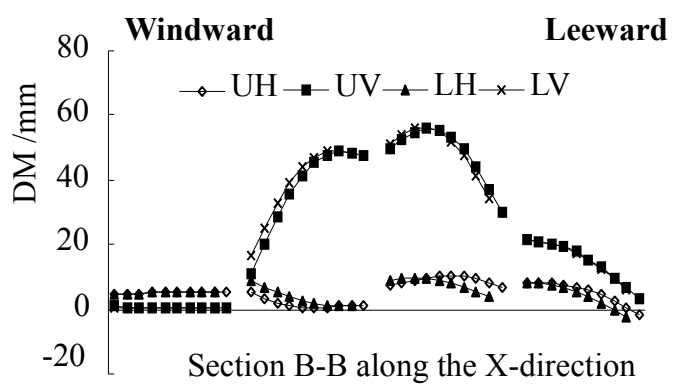

Figure 14. The Displacement Means (DM) of Various Nodes in the Substructures along Section B-B

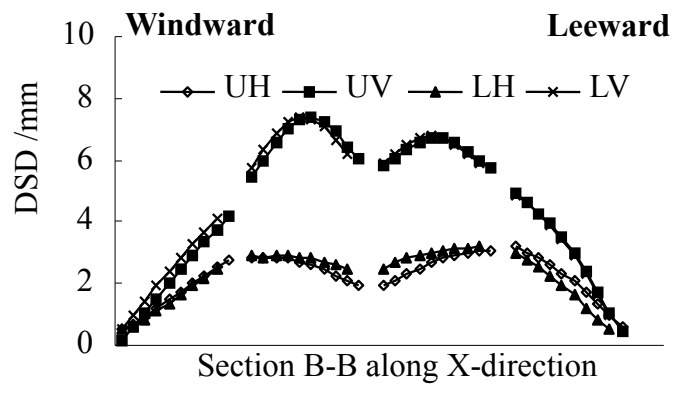

Figure 15. The Displacement Standard Deviations (DSD) of Various Nodes in the Substructures along Section B-B

Half of the structure is considered for the structural symmetry to investigate the nodal displacement distribution laws of the substructures with the same height along the longitudinal direction. The variation laws of the means and standard deviations of wind-induced nodal displacements of the substructures along the longitudinal section 3-3 are shown in Figures 16 and 17, respectively. It can be seen that, the magnitudes and distribution laws of nodal displacements of various substructures with same height are nearly identical along the longitudinal section. And the nodal displacements of the central substructures are slightly larger than those of the substructure at the end of the structure. Additionally, there is not remarkable variation in the nodal transverse horizontal displacements of each substructure along the longitudinal direction, while the nodal vertical displacements decrease gradually from the center to both sides of the substructure.

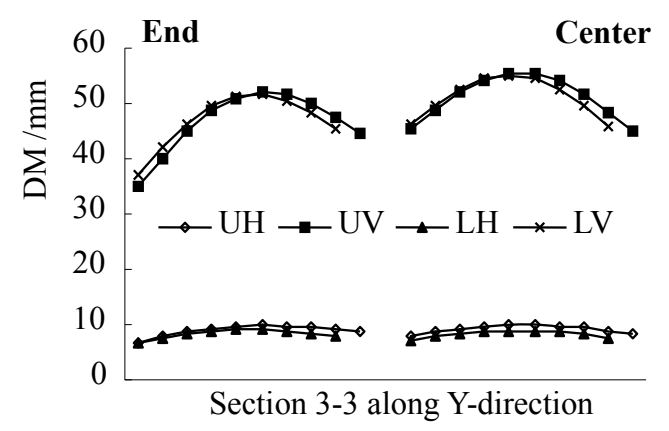

Figure 16. The DM of Various Nodes in the Substructures along Section 3-3

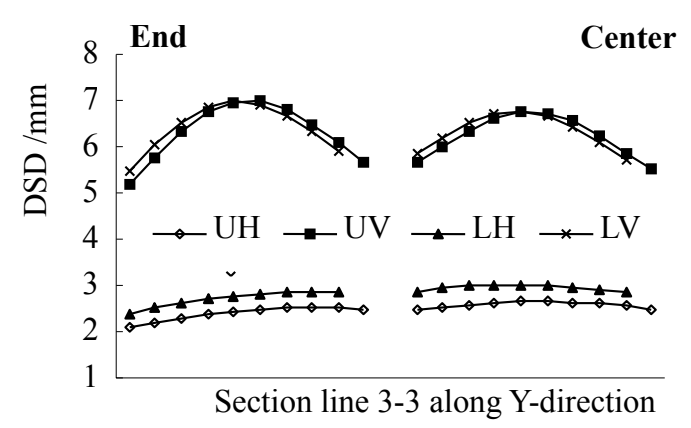

Figure 17. The DSD of Various Nodes in the Substructures along Section 3-3

\section{PARAMETRIC ANALYSES}

For the structure with given span, the wind-induced response behaviors must be different if different values of various parameters are taken. Thereby, parametric analyses are carried out in the this section. The standard deviations of the structural maximum wind-induced responses (including the maximum member internal force and nodal displacement) are taken as the indices to study the influence of various parameters on the wind-induced dynamic behaviors. And the structure with span of $80 \mathrm{~m}$ is still considered. When the influence of one parameter is investigated by changing its value to do analysis, the other parameters keep constant and identical with those described in Section 3.1. 


\subsection{Influence of the Structural Ratio of Rise to Span - $F$}

Consider different values of the structural ratio of rise to span $F$, such as $1 / 8,1 / 6,1 / 5,1 / 4$, and $1 / 3$, respectively. The relational curves of the wind-induced internal force and displacement versus $F$ are displayed in Figures 18 and 19, respectively.

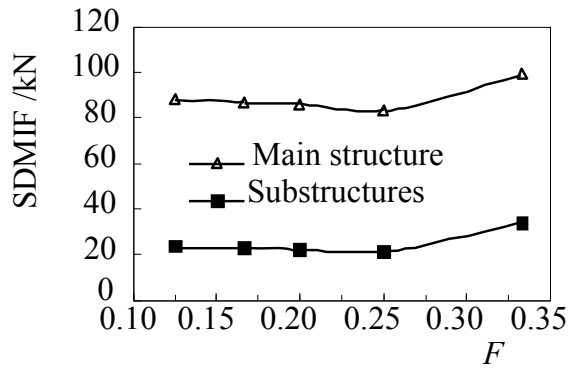

Figure 18. Relationship between the Standard Deviation of Maximum Internal Force (SDMIF) and the Ratio of Rise to Span of the Main Structure $-F$

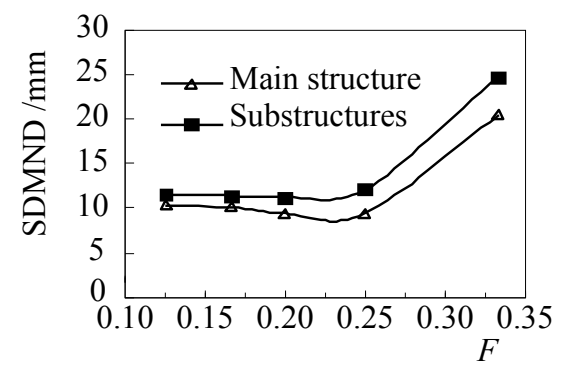

Figure 19. Relationship between the Standard Deviation of Maximum Nodal Displacement (SDMND) and the Ratio of Rise to Span of the Main Structure $-F$

It can be seen that, both the standard deviation of the maximum internal force and that of the maximum nodal displacement basically keep constant first but increase sharply when $F$ exceeds $1 / 4$ with increase of $F$. This indicates that the structural wind-induced dynamic effect will be very remarkable when the ratio of rise to span is large. Therefore, the structural ratio of rise to span should be no more than $1 / 4$ to keep good wind-resistant behavior.

\subsection{Influence of the Cross section of Members}

First, let the cross section of members in the substructures $S_{s}=\phi 76 \times 4.0$, and consider different cross sections of members in the main structure $S_{m}$ such as $\varphi 140 \times 4.5, \varphi 152 \times 5.0, \varphi 168 \times 5.5$, and $\varphi 180 \times 7.0$, respectively. Then, let $S_{m}=\phi 140 \times 4.5$, and take different values of $S_{s}$ such as $\phi 76 \times 4.0, \varphi 108 \times 4.5$, and $\varphi 140 \times 4.5$ to do analyses, respectively. The relational curves of the standard deviations of the maximum wind-induced internal force and displacement versus $S_{m}$ and $S_{s}$ are displayed in Figures 20, 21, 22, and 23, respectively.

It can be seen that, the standard deviation of the maximum wind-induced internal force of the main structure basically keeps constant and that of the substructures increases with increase of the cross section of members of the main structure, while the former increases and the latter basically keeps constant with increase of the cross section of members of the substructures. However, the standard deviation of the maximum wind-induced displacements of both the main structure and substructures decrease with increase in the cross section of members of whether the main structure or the substructures. On the whole, the structural rigidity can be enhanced by increase of the cross section of members, and the wind-resistant behavior of the structure can be improved, but the dynamic effect of member internal forces will also increase to a certain degree. Therefore, the increase of cross section of members should be moderate. 


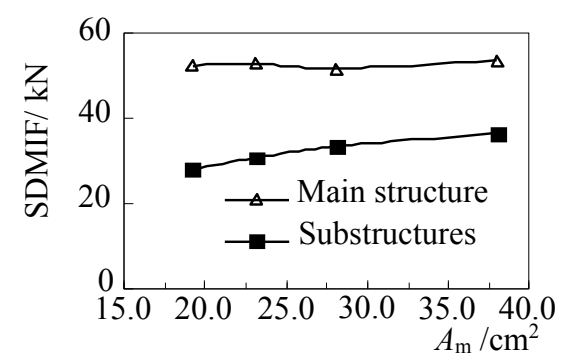

Figure 20. Relationship between the SDMIF and Sectional Area of Members in the Main Structure $-A_{\mathrm{m}}$

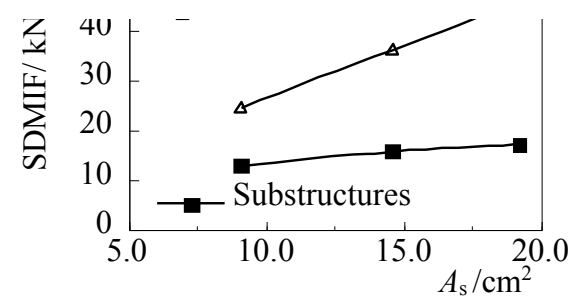

Figure 22. Relationship between the SDMIF and Sectional Area of Members in the Substructures $-A_{\mathrm{s}}$

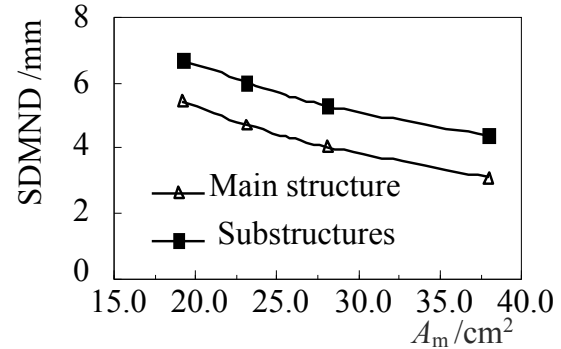

Figure 21. Relationship between the SDMND and Sectional Area of Members in the Main Structure $-A_{\mathrm{m}}$

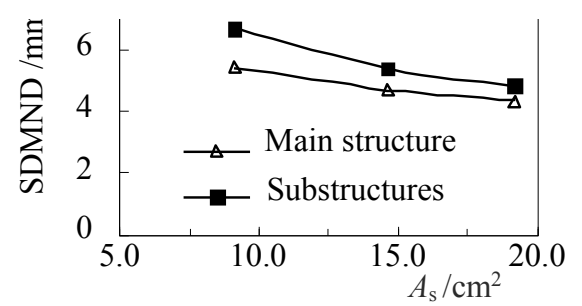

Figure 23. Relationship between the SDMND and Sectional Area of Members in the substructure $-A$ s

\subsection{Influence of the Damping Ratio}

In this section, different values of the damping ratio such as $0.01,0.02,0.03,0.04$, and 0.05 are considered, respectively. The relational curves of the standard deviations of the maximum wind-induced internal force and displacement with the damping ratio are displayed in Figures 24 and 25 , respectively. It can be seen that both the standard deviation of the maximum wind-induced internal force and that of the maximum displacement decrease with increase of the damping ratio, but the magnitude of decrease is very small. Additionally, the variation in damping ratio of steel lattice shell structure is actually inappreciable. Therefore, the influence of change in damping ratio on structural wind-induced dynamic behavior can be basically ignored.

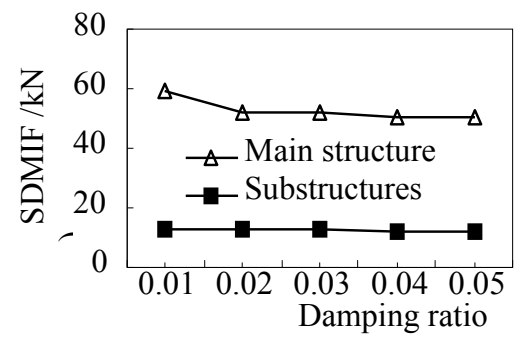

Figure 24. Relationship between the SDMIF and Damping Ratio

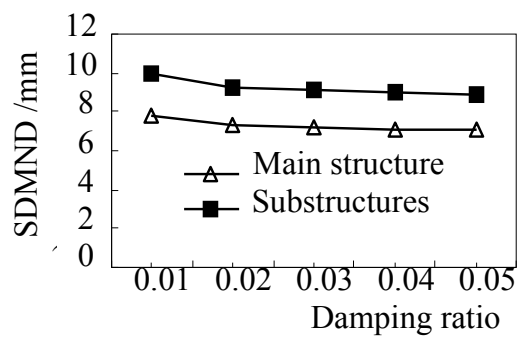

Figure 25. Relationship between the SDMND and Damping Ratio

\subsection{Influence of the Load}

Different values of the vertical uniform applied load such as $0.2,0.5,1.0,2.0$, and $3.0 \mathrm{kN} / \mathrm{m}^{2}$, are considered in this section. The relational curves of the standard deviations of the maximum wind-induced internal force and displacement with the applied load are displayed in Figures 26 and 
27 , respectively. It can be seen that, the standard deviation of the maximum internal force and that of the maximum displacement slightly with increase of the applied load. That is, the fluctuating effect of structural wind-induced response increases slightly with increase of the applied load. Thus, for this kind of structure, the variation of the roof covering material has small influence on the structural wind-induced dynamic behavior.

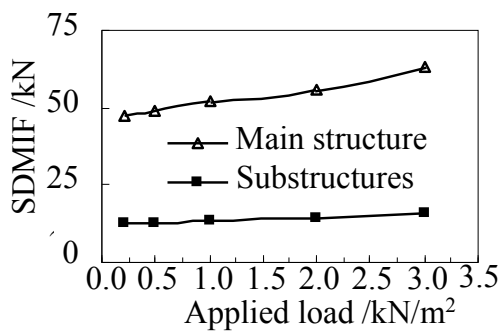

Figure 26. Relationship between the SDMIF and Applied Load

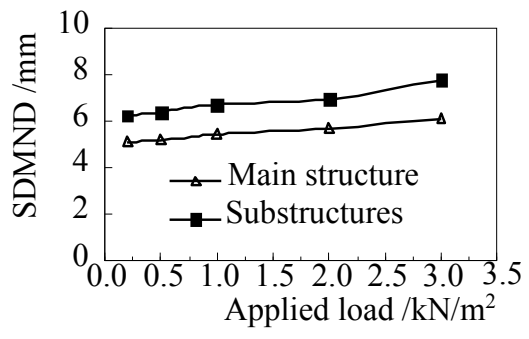

Figure 27. Relationship between the SDMND and Applied Load

\subsection{Influence of the Mean Wind Velocity}

Consider different standardized wind velocities such as $20,25,30$, and $35 \mathrm{~m} / \mathrm{s}$, respectively. The relational curves of the standard deviations of the maximum wind-induced internal force and displacement with the mean wind velocity are displayed in Figures 28 and 29, respectively. Obviously, the fluctuating effect of the wind-induced internal force and displacement increases linearly with increase of the mean wind velocity. That is, the magnitude of mean wind velocity has notable influence on dynamic effect of the structural wind-induced response.

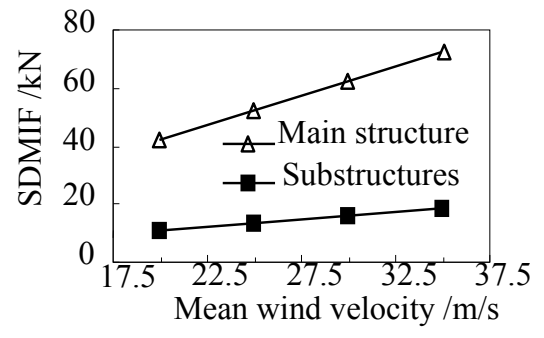

Figure 28. Relationship between the SDMIF and Mean Wind Velocity

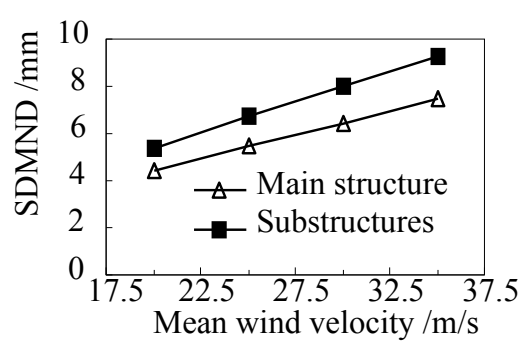

Figure 29. Relationship between the SDMND and Mean Wind Velocity

\subsection{Influence of the Support Conditions}

Different support styles are taken into consideration in this section, and the standard deviations of the maximum wind-induced internal forces and displacements under different support conditions are summarized in Table 1. In the table, the support styles B1 and B2 denote that the fixed-hinged supports are set on the lower and upper chord joints of the main nodes along the two longitudinal edges of the main structure, respectively; B3 presents that the fixed-hinged supports are set on both the upper and lower chord joints of the main nodes along the two longitudinal sides of the main structure; B4 means that the fixed-hinged supports are set on the lower chord joints of the main nodes along the four edges of the main structure; and B5 denotes that the fixed-hinged supports are set on the upper and lower chord joints of the main nodes along the two longitudinal edges and the lower chord joints of the main nodes along the two ends of the main structure. The details of various support styles were described in our previous work (He and Zhou [2]). 
Table 1. The Wind-induced Responses of the Structures under Different Support Conditions

\begin{tabular}{|c|c|c|c|c|c|c|}
\hline \multicolumn{2}{|c|}{ Support style } & $\mathrm{B} 1$ & $\mathrm{~B} 2$ & B3 & B4 & B5 \\
\hline \multirow{4}{*}{$\begin{array}{c}\text { The standard deviation of } \\
\text { maximum member internal } \\
\text { force } / \mathrm{kN} \\
\text { The standard deviation of } \\
\text { maximum nodal displacement } \\
\text { /mm }\end{array}$} & In the main structure & 61.613 & 61.074 & 45.894 & 49.463 & 66.509 \\
\hline & In the substructures & 14.744 & 15.250 & 14.656 & 13.868 & 19.726 \\
\hline & In the main structure & 8.780 & 10.122 & 6.372 & 7.638 & 8.248 \\
\hline & In the substructures & 10.164 & 10.420 & 7.940 & 8.960 & 11.003 \\
\hline
\end{tabular}

It can be seen that, the dynamic responses of the structure with support style B4 are somewhat smaller than those with support style B1. That is, when the fixed-hinged supports are only set on the lower chord joints of the main nodes along the two longitudinal edges of the main structure, the setting of supports on the lower chord joints of the main nodes along the two ends of the main structure can slightly improve the structural wind-resistant behavior. However, comparing styles B3 with B5 indicates that, when the fixed-hinged supports are set on the upper and lower chord joints of the main nodes along the two longitudinal edges of the main structure, the supports set along the two ends of the main structure will cause not improvement but degeneration of the structural wind-resistant behavior remarkably. Therefore, style B3, in which the fixed-hinged supports are set on the upper and lower chord joints of the main nodes along the two longitudinal edges of the main structure, is the best arrangement of supports for good wind-resistant behavior of the structure. And the second one is the case that the fixed-hinged supports are set on the lower chord joints of the main nodes along the four sides of the main structure. Apparently, the support styles B1, B2, and B5 are all not good for wind-resistance of the structure for either overabundance or insufficiency of the supports.

\section{ANALYSIS OF WIND-INDUCED VIBRATION COEFFICIENT}

The wind load is composed of the mean and fluctuating components. The response to the mean wind can be determined by static analytical method, which is relatively simple. However, the fluctuating wind is stochastic dynamic load, and the structural response should be solved by stochastic dynamic theory, which is obviously very complicated. In practical engineering, people always hope that the structural dynamic response can be dealt by static analytical method. Therefore, an equivalent static magnification coefficient, i.e. wind-induced vibration coefficient, should be introduced. Then the resultant response may be expressed as the product of the mean response and the wind-induced vibration coefficient.

The wind-induced vibration coefficients introduced in the current design code are determined based on the wind-induced vibration characteristics of high-rise structures, whose vibration displacements mainly depend on the first-order vibration mode. However, for a long span spatial structure, its natural frequency distribution is dense, and the structural response is not simply linear with the load. Therefore, the wind-induced vibration coefficient distribution in the structure is generally comparatively complicated.

Many scholars have studied the calculation method of wind-induced vibration coefficient of long-span spatial structures, and the wind-induced vibration coefficient was proposed based on responses, which was defined as the ratio of the possible maximum wind-induced response to the mean response of the structure in the wind duration (Chen et al. [23] and Uematsu et al. [24]). Also, the wind-induced vibration coefficient of element internal force and that of nodal displacement are commonly adopted. The wind-induced vibration coefficient of internal force $\beta_{f i}$ of any element $i$ and that of displacement $\beta_{d j}$ of any node $j$ can be expressed as follows. 


$$
\begin{aligned}
& \beta_{f i}=1+\mu \frac{\sigma_{f i}}{u_{f i}} \\
& \beta_{d j}=1+\mu \frac{\sigma_{d j}}{u_{d j}}
\end{aligned}
$$

in which, $u_{f i}$ and $\sigma_{f i}$ are the mean and standard deviation of wind-induced internal force of element $i$, respectively; $u_{d j}$ and $\sigma_{d j}$ are those of wind-induced displacement of node $j ; \mu$ is the peak factor.

The method has rationality, but it is not convenient for being used in practical engineering since the wind-induced vibration coefficients are very numerous. Statistic result shows that, the fluctuating component corresponding to the large wind-induced response is generally relatively large. Figures 30 and 31 depict the relationship of the standard deviation with the maximum and mean of the wind-induced internal force of each member, respectively.

It can be seen that, the standard deviation, i.e., the fluctuating component of the wind-induced member internal force is nearly linear with the maximum and mean values, and the same as the characteristics of the nodal displacement response. This is also illustrated by the similarity in distribution patterns of the means and corresponding standard deviations of wind-induced responses as shown in Figures 2-17. Therefore, the difference of the wind-induced vibration coefficients at different places in a structure given by Eq. 2 or 3 is not very remarkable. The concrete data are omitted. Accordingly, a singe wind-induced vibration coefficient can thus be adopted to reflect the whole wind-induced vibration character of this kind of structure except for several singular points.

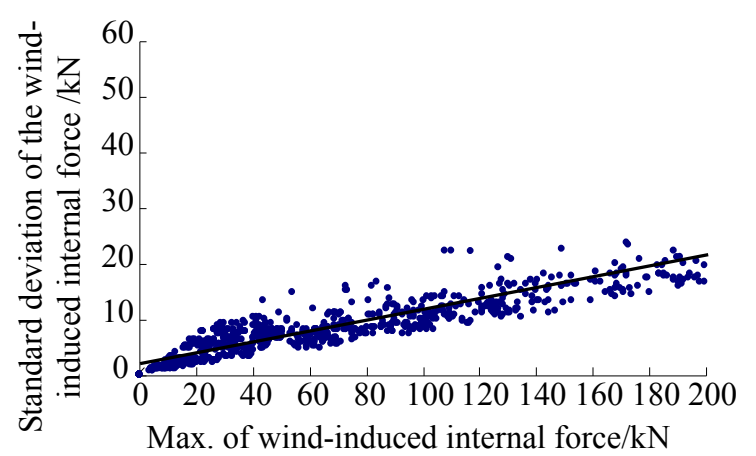

Figure 30. Relationship between the Standard Deviation and Max. of the Wind-induced Internal Force of Member

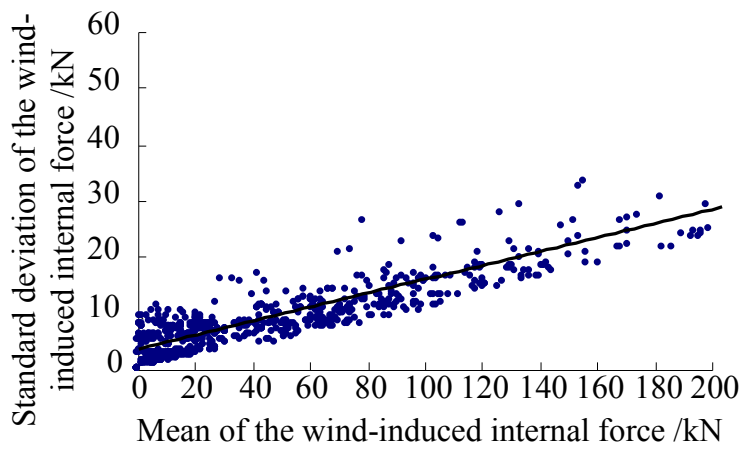

Figure 31. Relationship between the Standard Deviation and Mean of the Wind-induced Internal Force of Member

Results show that some wind-induced vibration coefficients may be too large, those are so-called singular points. The main reason is that the means of the responses are small, while the corresponding standard deviations of the fluctuating responses are relatively large. Actually, the resultant responses of these nodal displacements or element internal forces are generally comparatively small, so they are not the governing factors though their wind-induced vibration coefficients are large. Obviously, it is the peak nodal displacements and member internal forces of the structure that play a dominant role in design. Hence, for the structural governing wind-induced vibration coefficient $\beta$ can be defined as follows based on the envelope idea, 
$\beta=\frac{(u)_{\max }+\mu(\sigma)_{\max }}{(u)_{\max }}=1+\mu \frac{(\sigma)_{\max }}{(u)_{\max }}$

in which, $(\sigma)_{\max }$ and $(u)_{\max }$ are the maximum standard deviation and mean of various responses, and the peak factor $\mu=3.5$ (Lu et al. [25])。

The formula of the governing wind-induced vibration coefficient depends on the maximum standard deviation and mean of various responses, and it is reasonable to take it as the single design wind-induced vibration coefficient to avoid the inconvenience of numerous wind-induced vibration coefficients. Actually, this governing wind-induced vibration coefficient is safe in structural design, since the standard deviation corresponding to the maximum mean response is not always just the maximum one and thus the calculated maximum resultant response $(u)_{\max } \cdot \beta=(u)_{\max }+\mu(\sigma)_{\max }$ will be larger than the actual value.

Based on above discussion, the governing wind-induced vibration coefficient of the cylindrical reticulated mega-structures given by Eq. 4 is investigated in detail in this section based on large numbers of numerical examples with different parameters. The results show that, the displacement or internal force governing wind-induced vibration coefficient increases first and then decreases gradually with increase of the ratio of rise to span, and reaches the maximum value when the ratio of rise to span is $1 / 5$. It also increases gradually with increase of the structural span and the average wind velocity. Nevertheless, the governing wind-induced vibration coefficients of the structures with different parameters basically keep between 1.4 and 1.7 . Therefore, it can be safely taken as 1.5-1.8 to simplify the design process in practical engineering.

\section{CONCLUSION}

The wind-induced responses of the cylindrical LITDBS reticulated mega-structure braced with plane-plate grid substructures are studied by time history method in this paper. The basic distribution laws of the wind-induced internal forces and displacements of both the main structure and substructures are first analyzed. Then the influence of various parameters on the wind-induced responses is investigated in detail. Finally the structural wind-induced vibration coefficient is studied. The main conclusions can be drawn as follows.

(1) The upper and lower chord members of various latticed 3D arches are the main load-carrying elements. The largest wind-induced internal forces appear in the lower chord members of the bottom of the arches on the leeward side of the structure. Additionally, the intersection areas of the latticed 3D arches and longitudinal beams are the main load-carrying places of the main structure, to which more attention should be paid in design. Also, the wind-induced internal forces of the substructures are far smaller than those of the main structure, and the transverse upper chord members are the main stressed elements of the substructures.

(2) The main governing displacement in wind-resistance design exists on the top of the arches, and the peak displacement of the substructures is slightly larger than that of the main structure under the action of wind. Moreover, the distribution of the wind-induced nodal displacements, whose main component is the vertical one, is relatively uniform.

(3) The structural wind-induced dynamic effect will be very remarkable when the ratio of rise to span is large, and the structural ratio of rise to span should not be more than $1 / 4$ to keep good wind-resistant behavior. The variation of the dead load and mean wind velocity has small influence on the structural wind-induced dynamic behavior, but the impact of change in damping ratio on it can be basically ignored. 
(4) The structural rigidity can be enhanced by increase of the cross section of members, and the wind-resistant behavior of the structure can be improved, but the dynamic effect of member internal forces will also increase to a certain degree. Therefore, the increase of cross section of members should be moderate. The best arrangement of supports for good wind-resistant behavior of the structure is to set the fixed-hinged supports on the upper and lower chord joints of the main nodes along the two longitudinal edges of the main structure.

(5) The governing wind-induced vibration coefficient of this kind of structure basically keeps between 1.4 and 1.7, and it can be safely taken as 1.5-1.8 in design of practical engineering.

\section{ACKNOWLEDGEMENTS}

This work was financially supported by the Doctoral Fund of Ministry of Education of China (Grant no. 20120161110019), National Natural Science Foundation of China (Grant no. 51178176), Natural Science Foundation of Hunan Province (Grant no. 13JJ6022), Science and Technology Innovative Research Team Project of Universities in Hunan Province, and the Fundamental Research Funds for the Central Universities of Hunan University, which are gratefully acknowledged.

\section{REFERENCES}

[1] Makowski, Z.S., "Space Structure-A Review of Development in Last Decade", Space Structure IV, London, 1993, pp. 2-15.

[2] He, Y.J. and Zhou, X.H., "Static Properties and Stability of Cylindrical ILTDBS Reticulated Mega-structure with Double-layer Grid Substructures", Journal of Constructional Steel Research 2007, Vol. 63, No. 12, pp. 1580-1589.

[3] Zhou, X.H., He, Y.J. and Xu, L., "Formation and Stability of a Cylindrical ILTDBS Reticulated Mega-structure Braced with Single-layer Latticed Membranous Shell Substructures", Thin-Walled Structures, 2009, Vol. 47, No. 5, pp. 537-546.

[4] Lu, C.L., Li, Q.S., Huang, S.H., Chen, F.B. and Fu, X.Y., "Large Eddy Simulation of Wind Effects on a Long-span Complex Roof Structure", Journal of Wind Engineering and Industrial Aerodynamics, 2012, Vol. 100, pp. 1-18.

[5] Mehta, K.C., "Wind Load Standards", Wind Effects on Buildings and Structures-Proceedings of Jubileum Conference on Wind Effects on Building and Structures, Porto Alegre, Brazil, 1998, pp. 307-313.

[6] Chen, B., Wu, Y. and Shen, S.Z., "A New Method for Wind-induced Response Analysis of Long Span Roofs", International Journal of Space Structures, 2006, Vol. 21, No. 2, pp. 93-101.

[7] Oskoei, S.A.G. and McClure, G., "Dynamic Analysis of Cable Roofs under Transient Wind: A Comparison between Time Domain and Frequency Domain Approaches", Tsinghua Science \& Technology, 2008, Vol. 13, No. S1, pp. 53-57.

[8] Soares, Jr. D., and Mansur, W.J., "A Frequency-domain FEM Approach Based on Implicit Green's Functions for Non-linear Dynamic Analysis", International Journal of Solids and Structures, 2005, Vol. 42, No. 23, pp. 6003-6014.

[9] Uematsua, Y., Kuribaraa, O., Yamadab, M., et al., "Wind-induced Dynamic Behavior and Its Load Estimation of a Single-layer Latticed Dome with a Long Span”, Journal of Wind Engineering and Industrial Aerodynamics, 2001, Vol. 89, No. 14-15, pp. 1671-1687.

[10] Thai, H.T. and Kim, S.E., "Nonlinear Inelastic Time-history Analysis of Truss Structures", Journal of Constructional Steel Research, 2011, Vol. 67, No. 12, pp. 1966-1972. 
[11] Trombetti, T. and Silvestri, S., "On the Modal Damping Ratios of Shear-type Structures Equipped with Rayleigh Damping Systems", Journal of Sound and Vibration, 2006, Vol. 292, No. 1-2, pp. 21-58.

[12] Zhang, Z.Q., Chen, Y., Su, G.X. and Zhu, X.G., "Analysis of Wind-induced Vibration Control of Long-span Space Structure", Advanced Materials Research, 2011, Vol. 255-260, pp.1204-1208.

[13] Masters, F., Gurley, K., and Kopp, G.A., "Multivariate Stochastic Simulation of Wind Pressure over Low-rise Structures through Linear Model Interpolation", Journal of Wind Engineering and Industrial Aerodynamics, 2010, Vol. 98, No. 4-5, pp. 226-235.

[14] Karmakar, D., Ray-Chaudhuri, S. and Shinozuka, M., "Conditional Simulation of Non-Gaussian Wind Velocity Profiles: Application to Buffeting Response of Vincent Thomas Suspension Bridge”, Probabilistic Engineering Mechanics, 2012, Vol. 29, pp. 167-175.

[15] Owen, J.S., Eccles, B.J., Choo, B.S. et al. "The Application of Auto-regressive Time Series Modeling for the Time-frequency Analysis of Civil Engineering Structures”, Engineering Structures, 2001, Vol. 23, No. 5, pp. 521-536.

[16] Soares, D. and Mansur, W.J., "A Time Domain FEM Approach based on Implicit Green's Functions for Non-linear Dynamic Analysis", International Journal for Numerical Methods in Engineering, 2005, Vol. 62, No. 5, pp. 664-681.

[17] Kim, S.E., Kim, Y. and Choi, S.H., "Nonlinear Analysis of 3D Steel Frames", Thin-Walled Structures, 2001, Vol. 39, No. 6, pp. 445-61.

[18] Davenport, A.G., "The Relationship of Wind Structure and Wind Loading”, Proceeding of the Symposium on Wind Effect on Building and Structures, Vol. 1, London, 1965, pp. 54-102.

[19] Iwatani, Y., "Simulation of Multidimensional Wind Fluctuations having any Arbitrary Power Spectra and Cross Spectra", Journal of Wind Engineering, 1982, Vol. 11, pp. 5-18 [in Japanese].

[20] Isyumov, N., "Alan G. Davenport's Mark on Wind Engineering”, Journal of Wind Engineering and Industrial Aerodynamics, 2012, Vol. 104-106, pp. 12-24.

[21] He, Y.J., Zhou, X.H. and Wang, H.S., "Numerical Simulation of Wind Speed on Super-long-span Roof Structures", Journal of Hunan University (Natural Sciences), 2008, Vol. 35, No. 9, pp. 1-5 [in Chinese].

[22] Jakobsen, J.B., Andersen, T.L., Macdonald, J.H.G., Nikitas, N., Larose, G.L., Savage, M.G. and McAuliffe, B.R., "Wind-induced Response and Excitation Characteristics of an inclined Cable Model in the Critical Reynolds Number Range", Journal of Wind Engineering and Industrial Aerodynamics, 2012, Vol. 110, pp. 100-112.

[23] Chen, F.B., Li, Q.S., Wu, J.R. and Fu, J.Y., "Wind Effects on a Long-span Beam String Roof Structure: Wind Tunnel Test, Field Measurement and Numerical Analysis", Journal of Constructional Steel Research, 2011, Vol. 67, No. 10, pp. 1591-1604.

[24] Uematsu, Y., Yamada, M., Inoue, A. and Hongo, T., "Wind loads and Wind-induced Dynamic Behavior of a Single-layer Latticed Dome", Journal of Wind Engineering and Industrial Aerodynamics, 1997, Vol. 66, No. 3, pp. 227-248.

[25] Lu, F., Lou, W.J. and Sun, B.N., "Wind-induced Dynamic Response and Wind Load Factor for Long-span Flat Roof Structures”, Engineering mechanics, 2002, Vol. 19, No. 2, pp. 52-57 [in Chinese]. 